\title{
Localization of atomic ensembles via superfluorescence
}

\author{
Mihai Macovei, ${ }^{*}$ Jörg Evers, ${ }^{\dagger}$ and Christoph H. Keitel ${ }^{\ddagger}$ \\ Max-Planck-Institute for Nuclear Physics, Saupfercheckweg 1, D-69117 Heidelberg, Germany \\ M. Suhail Zubairy ${ }^{\S}$ \\ Institute for Quantum Studies and Department of Physics, Texas A\&M University, College Station, Texas 77843, USA; \\ Max-Planck-Institute for Nuclear Physics, Saupfercheckweg 1, D-69117 Heidelberg, Germany; \\ and Texas A\&M University at Qatar, Education City, P. O. Box 23874, Doha, Qatar
}

(Received 14 November 2006; published 2 March 2007)

\begin{abstract}
The subwavelength localization of an ensemble of atoms concentrated to a small volume in space is investigated. The localization relies on the interaction of the ensemble with a standing wave laser field. The light scattered in the interaction of the standing wave field and the atom ensemble depends on the position of the ensemble relative to the standing wave nodes. This relation can be described by a fluorescence intensity profile, which depends on the standing wave field parameters and the ensemble properties and which is modified due to collective effects in the ensemble of nearby particles. We demonstrate that the intensity profile can be tailored to suit different localization setups. Finally, we apply these results to two localization schemes. First, we show how to localize an ensemble fixed at a certain position in the standing wave field. Second, we discuss localization of an ensemble passing through the standing wave field.
\end{abstract}

DOI: $10.1103 /$ PhysRevA.75.033801

PACS number(s): 42.50.Fx, 32.50. $+\mathrm{d}, 32.80 .-\mathrm{t}$

\section{INTRODUCTION}

Nanotechnology requires an accurate control of the interacting components, in terms of both detection and preparation. This is a major motivation for the considerable attention that was devoted recently to subwavelength localization of single particles. Several remarkable schemes were proposed to achieve this goal [1-8]. In these schemes, typically the interaction of the object with the measurement fields serves two purposes. First, if the unknown initial position probability distribution of the object is phase coherent, then the interaction with the measurement fields localizes the object in the quantum mechanical sense, rather than providing a simple classical position measurement [8]. Second, after the localization, the measurement provides a signature of the position of the particle. Of related interest is the problem of localizing and distinguishing two nearby particles. Optical resolution of two molecules at the nanometer scale and manipulation of their degree of entanglement was experimentally demonstrated in [9], and the collective interaction between pairs of oriented nanostructures was considered in [10]. Also the measurement of the relative position of two atoms assisted by spontaneous emission [11] and the measurements of interparticle separations on a scale smaller than the emission wavelength [12] were investigated in detail. Somewhat related, considerable effort is devoted to quantum lithography $[13,14]$. The above localization schemes, however, have in common that they apply to the localization of a

\footnotetext{
*On leave from Technical University of Moldova and Institute of Applied Physics, Chişinău, Moldova. Electronic address: mihai.macovei@mpi-hd.mpg.de

†Electronic address: joerg.evers@mpi-hd.mpg.de

"Electronic address: keitel@mpi-hd.mpg.de

${ }^{\S}$ Electronic address: zubairy@physics.tamu.edu
}

single particle or to the measurement of relative position of two individual particles. In many cases of interest, however, an ensemble of particles is concentrated to a small region in space such that the ensemble properties become relevant, while the properties of the individual ensemble constituents cannot be resolved or are rapidly fluctuating in time.

Therefore, here we describe a scheme capable of localizing an ensemble of two-level atoms which are bunched together in a volume much smaller than an emission wavelength. Possible realizations include small clusters, few-atom impurities, or atoms trapped, e.g., in optical lattices. The localization relies on the coherent interaction with a standing wave electromagnetic field. Since the interatomic distances are small, the atoms interact collectively via the environmental vacuum modes. One consequence of this is the appearance of superfluorescence; i.e., the scattered light intensity scales with the number of atoms, $N$, squared $\left(I \propto N^{2}\right)$. We find that the fluorescence light emitted collectively by the ensemble is a function of the ensemble position in the standing wave. In particular, for suitable standing wave parameters and for ensemble positions around the nodes of the standing wave field, the emitted fluorescence intensity sharply drops to a minimum over a narrow spatial region. The narrow width of the dip in the spatial intensity profile is a direct consequence of the collectivity. Our scheme is different from localization schemes discussed previously, as here the localization relies on the absence of the fluorescence light, rather than its properties. The spatial resolution is determined by the width of the dip in the intensity profile. Since this collective fluorescence intensity profile is our main observable, we discuss the profile in detail in terms of the available free parameters and show that the profile can be tailored to suit a given localization setup. Based on these results, we then propose two schemes which exploit this spatial fluorescence intensity profile to localize an ensemble of atoms. First, we assume the sample to be fixed within the standing wave field. 
In this case, the spatial fluorescence intensity profile can be scanned along the standing wave axis by changing the relative phase of the laser fields forming the standing wave. A continuous measurement of the intensity of the scattered light throughout this scan reveals the position of the sample on a subwavelength scale. We further show that this setup also enables one to measure the distance between two samples, the number of atoms in a sample, or the linear dimension of the sample. Second, we consider an atom cluster flying through the standing wave field. Here a scanning is not possible due to the short interaction time of ensemble and standing wave field. Rather, the absolute intensity of the scattered light can be used to recover the crossing position of the ensemble.

The article is organized as follows. In Sec. II, we present our theoretical model for the ensemble interaction with the standing wave field and derive the intensity profile as our main observable. Section III consists of three parts. In the first part, Sec. III A, we in detail study the collective fluorescence profile numerically. In Sec. III B, the results are applied to the localization of an ensemble fixed in the standing wave field. The last part, Sec. III C, discusses the localization of an ensemble flying through the standing wave field. Finally, Sec. IV discusses and summarizes the results.

\section{THEORY}

In the usual mean-field, dipole, and rotating wave approximations the interaction of such an atomic sample with an external laser field and the surrounding vacuum modes, in a frame rotating with laser frequency $\omega_{L}$, is described by the Hamiltonian $H=H_{0}+H_{L}+H_{I}$ where

$$
\begin{gathered}
H_{0}=\sum_{k} \hbar\left(\omega_{k}-\omega_{L}\right) a_{k}^{\dagger} a_{k}+\sum_{j=1}^{N} \hbar\left(\omega_{0 j}-\omega_{L}\right) S_{z j}, \\
H_{L}=\sum_{j=1}^{N} \hbar\left[\Omega\left(\vec{r}_{j}\right) S_{j}^{+}+\Omega^{*}\left(\vec{r}_{j}\right) S_{j}^{-}\right], \\
H_{I}=i \sum_{k} \sum_{j=1}^{N}\left(\vec{g}_{k} \cdot \vec{d}_{j}\right) a_{k}^{\dagger} S_{j}^{-} e^{-i \vec{k} \cdot \vec{r}_{j}}+\text { H.c. }
\end{gathered}
$$

Here $S_{j}^{ \pm}$are the raising and lowering operators for the $j$ th atom, positioned at $\vec{r}_{j}$ and obeying the commutation relations $\left[S_{j}^{+}, S_{l}^{-}\right]=2 S_{z j} \delta_{j l}$ and $\left[S_{z j}, S_{l}^{ \pm}\right]= \pm S_{j}^{ \pm} \delta_{j l}$ with $S_{z j}$ being the inversion operator. $a^{\dagger}$ and $a$ are the radiation creation and annihilation operators satisfying the commutation relations $\left[a_{k}, a_{k^{\prime}}^{\dagger}\right]=\delta_{k k^{\prime}}$ and $\left[a_{k}, a_{k^{\prime}}\right]=\left[a_{k}^{\dagger}, a_{k^{\prime}}^{\dagger}\right]=0$.

In Eq. (1), $H_{0}$ represents the free electromagnetic field (EMF) and free atomic Hamiltonians, respectively. The second term-i.e., $H_{L}$-describes the interaction of the atomic system with an external standing wave coherent field. In general, the Rabi frequencies of the atoms in a standing wave are position dependent since

$$
\Omega\left(\vec{r}_{j}\right)=\Omega_{j} \cos \left(\vec{k}_{L} \cdot \vec{r}_{j}\right),
$$

where $\Omega_{j}=\left(\vec{d}_{j} \cdot \vec{E}_{L}\right) / \hbar$ while $E_{L}=\left|\vec{E}_{L}\right|$ is the amplitude of the electromagnetic field intensity with a wave vector $\vec{k}_{L}$ and $\vec{d}_{j}$ is the dipole moments of the atoms. Note that the scheme described here can also be generalized to multiphoton transitions. Then, the Rabi frequency can be written as

$$
\Omega\left(\vec{r}_{j}\right)=\Omega_{j}^{(n)} \cos \left(n \vec{k}_{L} \cdot \vec{r}_{j}\right),
$$

where $\Omega_{j}^{(n)}$ is a multiphoton Rabi frequency arising from an adiabatic elimination of intermediate states and $n$ denotes the number of photons involved in the multiphoton process. In this way, the wavelength $\lambda=2 \pi / k$ can be reduced to the effective wavelength $\lambda / n$, thus increasing the spatial resolution [14]. The last term in Eq. (1), $H_{I}$, takes into account the interaction of all atoms with the environmental vacuum modes.

In the Born-Markov approximations the quantum dynamics of the driven multiatom sample (each atom having identical transition frequency $\omega_{0}$ ) is governed by the master equation [15-18]

$$
\begin{gathered}
\frac{d}{d t} \rho(t)+\frac{i}{\hbar}\left[\widetilde{H}_{0}, \rho\right]=-\sum_{i, j=1}^{N}\left\{\gamma_{i j}\left(\omega_{0}\right)\left[S_{j}^{+}, S_{l}^{-} \rho\right]+\text { H.c. }\right\}, \\
\widetilde{H}_{0}=\hbar \sum_{j}\left[\Delta S_{z j} / 2+\Omega\left(\vec{r}_{j}\right) S_{j}^{+}+\text {H.c. }\right] .
\end{gathered}
$$

Here, $\Delta=\omega_{0}-\omega_{L}$ is the detuning of atomic levels from the frequency of the driving field. Further,

$$
\gamma_{j l}\left(\omega_{0}\right)=\chi_{j l}\left(\omega_{0}\right)+i \Omega_{j l}\left(\omega_{0}\right),
$$

where the collective parameters describing the mutual interactions among any atomic pair in the sample are given, respectively, by $[15,16]$

$$
\begin{aligned}
\chi_{j l}(\omega)= & \frac{3 \gamma}{2}\left\{\left[1-\cos ^{2} \xi_{j l}\right] \frac{\sin \left(\omega r_{j l} / c\right)}{\omega r_{j l} / c}+\left[1-3 \cos ^{2} \xi_{j l}\right]\right. \\
& \left.\times\left[\frac{\cos \left(\omega r_{j l} / c\right)}{\left(\omega r_{j l} / c\right)^{2}}-\frac{\sin \left(\omega r_{j l} / c\right)}{\left(\omega r_{j l} / c\right)^{3}}\right]\right\}, \\
\Omega_{j l}(\omega)= & \frac{3 \gamma}{4}\left\{\left[\cos ^{2} \xi_{j l}-1\right] \frac{\cos \left(\omega r_{j l} / c\right)}{\omega r_{j l} / c}+\left[1-3 \cos ^{2} \xi_{j l}\right]\right. \\
& \left.\times\left[\frac{\sin \left(\omega r_{j l} / c\right)}{\left(\omega r_{j l} / c\right)^{2}}+\frac{\cos \left(\omega r_{j l} / c\right)}{\left(\omega r_{j l} l c\right)^{3}}\right]\right\},
\end{aligned}
$$

with $2 \gamma=4 \omega_{0}^{3} d_{0}^{2} /\left(3 \hbar c^{3}\right)$ being the single-atom spontaneous decay rate. Here, we have assumed that all the dipole moments are identical and parallel-i.e., $d_{j}=d_{l} \cdots \equiv d_{0}$-and then $\xi_{j l}$ is the angle between the dipole moments $\vec{d}_{0}$ and $\vec{r}_{j l}$ $=\vec{r}_{j}-\vec{r}_{l}$.

Inspecting the master equation (3) one can easily distinguish the part of it describing the coherent evolution of atoms under the influence of the laser field-i.e., the term containing the Hamiltonian $\widetilde{H}_{0}$-from that characterizing the collective spontaneous emission due to the vacuum modesthat is, the terms proportional to $\operatorname{Re}\left\{\gamma_{j l}\left(\omega_{0}\right)\right\}$. The dipoledipole interactions between the two-level atoms are described by the terms proportional to $\operatorname{Im}\left\{\gamma_{j l}\left(\omega_{0}\right)\right\}$. If the 
interparticle separations are small enough-that is, $\omega r_{j l} / c$ $\equiv k r_{j l} \rightarrow 0 \quad(j \neq l)$ - then to second order in this parameter, Eqs. (6) reduce to

$$
\begin{aligned}
\chi_{j l}(k)=\gamma\left\{1-\frac{1}{5}\left(k r_{j l}\right)^{2}\left[1-\frac{1}{2} \cos ^{2} \xi_{j l}\right]\right\}, \\
\Omega_{j l}(k)=3 \gamma\left\{\left[\cos ^{2} \xi_{j l}-1\right]\left(2 / k r_{j l}-k r_{j l}\right)+\left[1-3 \cos ^{2} \xi_{j l}\right]\right. \\
\left.\times\left[\left(k r_{j l}\right)^{-1}-k r_{j l} / 4+2\left(k r_{j l}\right)^{-3}\right]\right\} / 8 .
\end{aligned}
$$

It is easy to realize that in this case $\chi_{j l} \rightarrow \gamma$ while $\Omega_{j l}$ reduces to the static dipole-dipole interaction potential-i.e.,

$$
\Omega_{j l}=\frac{3 \gamma}{4\left(k r_{j l}\right)^{3}}\left\{1-3 \cos ^{2} \xi_{j l}\right\} .
$$

For lower atomic densities the collective parameters $\chi_{j l}$ and $\Omega_{j l}$ vanish because the atoms react independently from each other in this particular case. Finally, the master equation (3) describes adequately driven atomic samples of any shapes providing that retardation effects are negligible.

When dealing with smaller atomic systems of an arbitrary irregular shape it is hard to specify the orientation of dipole moments relative to the interparticle separations. That is to say, we do not have a privileged angular distribution of photons as they are emitted equally in all directions. Since there is no information on the dipole orientations, we average the dipole-dipole interaction potential over all directions. Interestingly, the static dipole-dipole interaction given by Eq. (8) vanishes in this case. Then, according to the second term in Eq. (7), the averaged dipole-dipole interactions among the two-level emitters are given by the expression

$$
\Omega_{j l}^{(a v)}=-\frac{\gamma}{2 k r_{j l}} .
$$

Thus, the dipole-dipole potential reduces from a short-range to a long-range interaction, although the radiators are close to each other. Moreover, the influence of the dipole-dipole interactions, in a two-atom system, was shown to be negligible in practice for interparticle separations such that $k r_{j l}$ $\geq \pi / 10$ [19].

In what follows we shall apply Eqs. (3)-(9) to the localization of a small atomic system within an emission wavelength. Suppose that the linear dimension of the atomic sample is much less than the emission wavelength (say, for instance, smaller than $0.1 \lambda$ ). Under this assumption the twolevel emitters are almost in an equivalent position relative to the driving standing wave laser and we can omit the atomic indices from the expression characterizing the Rabi frequency-i.e., $\Omega\left(r_{j}\right) \approx \Omega(r) \equiv \Omega(x)$. The master equation (3) transforms then into

$$
\begin{aligned}
& \frac{d}{d t} \rho(t)+i\left[\tilde{\Delta} S_{z}+\Omega(x)\left(S^{+}+S^{-}\right)-\Omega_{d} S^{+} S^{-}, \rho\right] \\
& \quad=-\gamma\left\{\left[S^{+}, S^{-} \rho\right]+\left[\rho S^{+}, S^{-}\right]\right\} .
\end{aligned}
$$

Here $\widetilde{\Delta}=\Delta+\Omega_{d}$ and $\left\{S^{ \pm}, S_{z}\right\}$ are collective atomic operators satisfying the standard commutation relations of $\mathrm{su}(2)$ algebra [15-18] while $\Omega_{d}$ is the dipole-dipole interaction poten- tial considered identical for all radiators. Note that Eq. (10) describes a small driven atomic system that involves symmetrized multiparticle states only. The antisymmetric states are decoupled from the dynamics within our current framework, and in the following we assume that they are not populated initially. The dipole-dipole interaction $\Omega_{d}$ considerably shifts the symmetric states from the field resonance if the interparticle separations are very small [20]. Even though the hypothesis of identical dipole-dipole interactions is, in general, not fulfilled, it admits to solve analytically the above master equation in the long-time limit. Thus, in order to get some insight into how the dipole-dipole interactions affect the localization processes of a small system as a whole we shall accept the hypothesis of identical dipole-dipole interactions between any pair in the sample.

The solving procedure of Eq. (10) was described in $[17,18]$ for running wave lasers. Adopting it to the case of a standing wave field, one arrives at the steady-state solution

$$
\rho_{s}=Z^{-1} \sum_{n, m=0}^{N} C_{n m}(x)\left(S^{-}\right)^{n}\left(S^{+}\right)^{m},
$$

where

$$
\begin{gathered}
C_{n m}(x)=(-1)^{n+m} \alpha^{-n}\left(\alpha^{*}\right)^{-m} a_{n m}, \\
a_{n m}=\frac{\Gamma(1+n+\beta) \Gamma\left(1+m+\beta^{*}\right)}{n ! m ! \Gamma(1+\beta) \Gamma\left(1+\beta^{*}\right)},
\end{gathered}
$$

with $\alpha=i \Omega(x) /\left(\gamma+i \Omega_{d}\right)$ and $\beta=i \widetilde{\Delta} /\left(\gamma+i \Omega_{d}\right)$. The normalization constant $Z$ is chosen such that $\operatorname{Tr}\left\{\rho_{s}\right\}=1-$ i.e.,

$$
Z=\sum_{n, m=0}^{N}(-1)^{n+m} \alpha^{-n}\left(\alpha^{*}\right)^{-m} a_{n m} \operatorname{Tr}\left\{\left(S^{-}\right)^{n}\left(S^{+}\right)^{m}\right\} .
$$

The trace can be performed using the relations

$$
\begin{aligned}
& S^{+}|s, l\rangle=\sqrt{(s-l)(s+l+1)}|s, l+1\rangle, \\
& S^{-}|s, l\rangle=\sqrt{(s+l)(s-l+1)}|s, l-1\rangle,
\end{aligned}
$$

where the collective Dicke states $|s, l\rangle$, with $s=N / 2$ and $-s$ $\leq l \leq s$, are the eigenstates for the operator $S_{z}$ and the operator of the total "spin" $S^{2}[18]$ :

$$
\begin{gathered}
S_{z}|s, l\rangle=l|s, l\rangle, \\
S^{2}|s, l\rangle=s(s+1)|s, l\rangle .
\end{gathered}
$$

Thus,

$$
\begin{aligned}
\operatorname{Tr}\left\{\left(S^{-}\right)^{n}\left(S^{+}\right)^{m}\right\} & =\sum_{l=-s}^{s}\left\langle l, s\left|\left(S^{-}\right)^{n}\left(S^{+}\right)^{m}\right| l, s\right\rangle \\
& =\delta_{n, m} \sum_{l=-s}^{s}\left\langle l, s\left|\left(S^{-}\right)^{n}\left(S^{+}\right)^{n}\right| l, s\right\rangle
\end{aligned}
$$

and, then, 


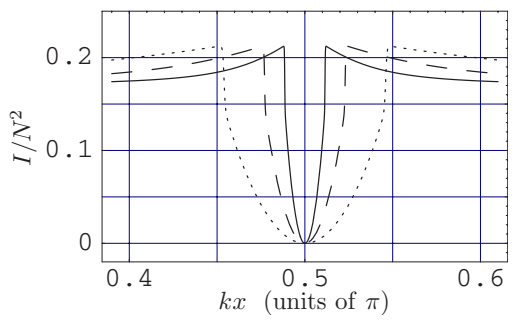

FIG. 1. (Color online) The dependence of the collective steadystate resonance fluorescence intensity $I / N^{2}$ as a function of $k x$. The solid, dashed, and dotted curves are for $\Omega /(N \gamma)=100,50$, and 25, respectively. Other parameters are $\Omega_{d} / \gamma=-10, \Delta /(N \gamma)=0.5$, and $N=100$.

$$
Z=\sum_{n=0}^{N} a_{n n}|\alpha|^{-2 n} \frac{(N+n+1) !(n !)^{2}}{(N-n) !(2 n+1) !} .
$$

The intensity of the collective resonance fluorescence emitted by driving the multiparticle system is calculated taking into account that this quantity is proportional to the firstorder atomic correlator-i.e., $I \propto\left\langle S^{+} S^{-}\right\rangle$. Then, using Eq. (11)-(14), one obtains

$$
I(x)=Z^{-1} \sum_{k=1}^{N} C_{k-1 k-1}(x) \frac{(N+k+1) !(k !)^{2}}{(N-k) !(2 k+1) !} .
$$

\section{RESULTS}

We now turn to the discussion of Eq. (15). First, we study the dependence of the collective fluorescence intensity on the various external parameters. Second, we show how the collective fluorescence intensity can be used to precisely locate a sample of particles which is fixed in space inside the standing wave field. Third, we discuss the localization of a collection of particles flying through the cavity field.

\section{A. Collective fluorescence intensity}

Figure 1 shows the collective fluorescence intensity versus the position of the multiparticle collection in the standing wave field. Note that $k x=\pi / 2$ corresponds to a node of the standing wave field, and thus the fluorescence intensity vanishes for particles located at this point in space. The parameters in Fig. 1 are the number of atoms, $N=100$, dipoledipole coupling constant $\Omega_{d} / \gamma=-10$, and detuning $\Delta /(N \gamma)$ $=0.5$. The solid line shows a standing wave Rabi frequency $\Omega /(N \gamma)=100$, the dashed line is for $\Omega /(N \gamma)=50$, and the dotted one is for $\Omega /(N \gamma)=25$. It can be seen that with decreasing Rabi frequency, the width of the dip in the fluorescence intensity around the node at $k x=\pi / 2$ increases. Thus a strong driving field allows for a narrow region in space that leads to vanishing fluorescence intensity, while a weaker field gives rise to fluorescence intensity over a wider range of positions.

The second free parameter is the detuning $\Delta$ between the driving field frequency and the bare transition frequency $\omega_{0}$ of the individual atoms in the sample. Figure 2 shows the

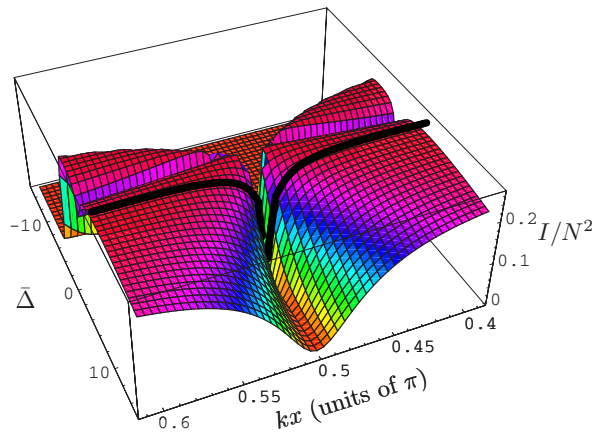

FIG. 2. (Color online) The steady-state collective resonance fluorescence intensity $I / N^{2}$ as a function of $k x$ and $\bar{\Delta}=\Delta /(N \gamma)$. Here $N=100, \Omega /(N \gamma)=50$, and $\Omega_{d} / \gamma=-10$. The black line on top of the surface plot indicates the position $\bar{\Delta}=\left|\Omega_{d}\right| / \gamma=10$.

dependence of the collective fluorescence intensity versus the position of the sample on this detuning. Note that the $y$ axis of this figure is a scaled detuning $\bar{\Delta}=\Delta /(N \gamma)$. Thus, $\bar{\Delta}$ $=0$ corresponds to the resonance case $\Delta=0$, whereas $\bar{\Delta}>0$ indicates $\Delta>0$ and thus $\omega_{L}<\omega_{0}$. A qualitative understanding of this figure can be gained from the case of a two-atom sample, as shown in Fig. 3. In a collective-state basis, the two-atom sample corresponds to a collective ground state $\left|g_{a}, g_{b}\right\rangle$ at energy 0 , where each of the two atoms $a$ and $b$ is in its respective ground state, an excited collective state $\left|e_{a}, e_{b}\right\rangle$ at energy $2 \hbar \omega_{0}$, and a symmetric [antisymmetric] collective state at energy $\hbar\left(\omega_{0}+\Omega_{d}\right)\left[\hbar\left(\omega_{0}-\Omega_{d}\right)\right]$ :

$$
\begin{aligned}
& |S\rangle=\frac{1}{\sqrt{2}}\left(\left|g_{a}, e_{b}\right\rangle+\left|e_{a}, g_{b}\right\rangle\right), \\
& |A\rangle=\frac{1}{\sqrt{2}}\left(\left|g_{a}, e_{b}\right\rangle-\left|e_{a}, g_{b}\right\rangle\right) .
\end{aligned}
$$

In the limit of small interparticle distance chosen in our analysis, the asymmetric state decouples from the dynamics, such that we are essentially left with a three-state ladder system. In Fig. 3, the symmetric state is located at $\bar{\Delta}=5$,

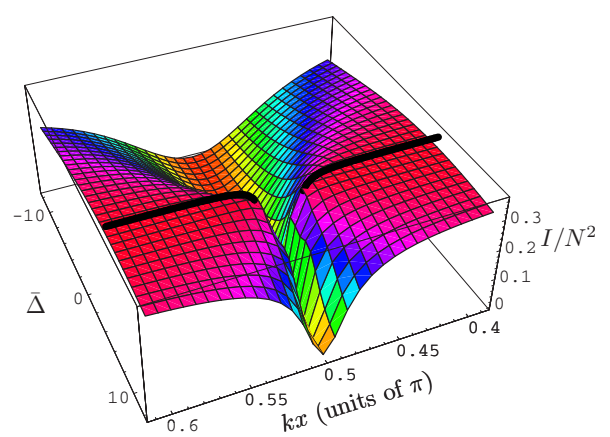

FIG. 3. (Color online) The steady-state collective resonance fluorescence intensity $I / N^{2}$ as a function of $k x$ and $\bar{\Delta}=\Delta /(N \gamma)$. Here $N=2, \Omega /(N \gamma)=50$, and $\Omega_{d} / \gamma=-5$. The black line on top of the surface plot indicates the position $\bar{\Delta}=5$ around which the twoatom symmetric collective state is located. 


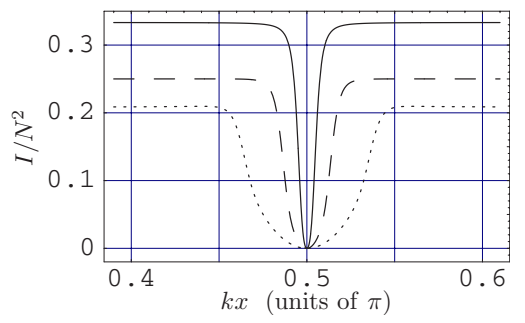

FIG. 4. (Color online) Collective fluorescence versus atom ensemble position in the standing wave field for different sizes of the ensemble. The parameters are $\Omega_{d} / \gamma=-5, \Delta / \gamma=10$, and $\Omega / \gamma=100$. The solid line is for the number of atoms, $N=2$, the dashed line shows the case $N=4$, and the dotted line is for $N=8$.

since the dipole-dipole coupling is chosen as $\Omega_{d}=-5 \gamma$. It can be seen that the width of the intensity dip is minimal if the driving field frequency is in resonance with the symmetric state and becomes wider in moving away from the resonance. No additional structure is visible around $\bar{\Delta}=-5$, where the asymmetric state is located, since it is decoupled. Note that for vanishing dipole-dipole interaction, $\Omega_{d}=0$, Figs. 2 and 3 would exhibit features symmetric with respect to both the planes given by $\bar{\Delta}=0$ and $k x=\pi / 2$.

These results from the two-atom case directly carry over to the many-particle sample. The minimum width of the intensity dip is close to the position where symmetric-state combinations can be expected, whereas no structure can be found towards asymmetric collective states. A direct identification of the position of the detuning with minimum dip width is difficult, however, since the collective-state basis of a multiparticle sample includes many symmetric collective states. In the example of Fig. 2, in a very small range around the node $k x=\pi / 2$ the dip at $\bar{\Delta}=10$ is narrowest, but its width increases faster in moving away from the node than it does for slightly lower values of $\bar{\Delta}$. The many-particle case also shows an additional structure at $\bar{\Delta}=0$ (see Fig. 2), which, however, is not of interest for our current localization scheme.

Finally, in Fig. 4, we show the dependence of the collective intensity on the number of particles in the sample. This is different from the previous results, since by changing the number of atoms, both the scaled Rabi frequency $\Omega /(N \gamma)$ and the scaled detuning $\Delta /(N \gamma)$ are changed at the same time. It can be seen from Fig. 4 that for a given standing wave intensity and a given detuning, varying the number of atoms in the ensemble changes the width of the intensity dip at the nodes. This can be understood by noting that a change of the number of atoms effectively shifts the position of the symmetric state resonance. Since the laser field frequencies are kept fixed in Fig. 4, this corresponds to moving along the $\bar{\Delta}$ axis in Figs. 2 and 3. Therefore, different widths of the intensity dip can be observed. The maximum intensity changes with $N$ in Fig. 4, since in this figure the unscaled Rabi frequency $\Omega$ is kept fixed. It should be noted that the parameters in Fig. 4 are such that for any shown number of atoms, the scaled Rabi frequency $\Omega /(N \gamma)$ dominates the dynamics. A further increase of the number of atoms which
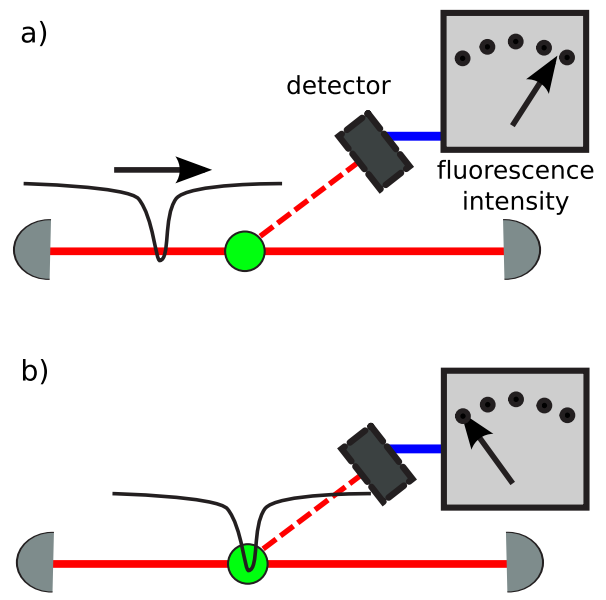

FIG. 5. (Color online) Scanning-dip scheme. The figure depicts a possible experimental implementation of our scheme. An atom ensemble (green dot) is assumed fixed inside the standing wave field. A detector measures the scattered fluorescence light, while the phase of the standing wave field is varied. The black curve symbolizes the fluorescence intensity profile as, e.g., shown in Fig. 1. If the intensity dip does not coincide with the ensemble position, then a high intensity of fluorescence is detected. But if the dip sweeps across the ensemble position, then the measured intensity drops to a minimum over a narrow spatial range, thus providing a subwavelength localization.

leads to $\left|\Omega_{d}\right| / \gamma \gg \Omega /(N \gamma)$ shifts the relevant collective states out of the laser field resonance such that the total fluorescence intensity vanishes.

\section{B. Scanning-dip spectroscopy}

After the discussion of the collective fluorescence intensity as our main observable, we now turn to the application of this observable to the localization of a collection of atoms. As the first setup, we consider a collection of atoms which is fixed inside the standing wave field at an unknown position. In order to detect the position of the sample, the total collective fluorescence intensity is continuously monitored, which may already provide a coarse position measurement. Then the relative phase of the two counterpropagating fields forming the standing wave is changed, such that the nodal structure of the field shifts along the standing wave propagation axis. Throughout this shift, the detected intensity is modulated in time with the collective fluorescence intensity profile, as depicted in Fig. 5. If the intensity profile is located such that its dip does not coincide with the actual position of the sample, then the intensity is near its maximum value; see Fig. 5(a). But if the two positions coincide, then the intensity vanishes; see Fig. 5(b). Obviously, for this scheme it is desirable to have the intensity dip as narrow as possible in order to achieve a localization well below the usual diffraction limit. According to our results of Sec. III A, this can be achieved by using a strong standing wave field and by tuning it close to the symmetric collective state resonance. For instance, the solid curve in Fig. 1 has a width of about $\Delta(k x)$ $=0.02 \pi$, corresponding to $\Delta x=0.01 \lambda$. Note, however, that the obtained accuracy is also limited by the spatial size of the 
ensemble if the dip width is smaller than the linear dimension of the sample.

The localization thus relies on the absence of the fluorescence intensity at the narrow intensity profile dip, as compared to previous localization schemes, which focused on the properties of the fluorescence light [1-12]. This has the advantage that if the ensemble is localized, few residual photons are scattered off the atoms and the perturbing effects of the light fields are small. Also, for a many-particle ensemble, the dipole-dipole interaction between the constituents is rather complicated, which renders the derivation of spatial information from the fluorescence light difficult. In our approach, no detailed knowledge of the dipole-dipole potential is required.

The present scheme can also be used to measure the distance between the center of masses of two ensembles by relating the required phase shifts between two positions with vanishing intensity to the measured intensity profile. Further, since the width of the intensity dip depends on the number of atoms in the ensemble if all other parameters remain fixed (see Fig. 4), this scheme can also be used to obtain the number of atoms in the ensemble located in the standing wave field by measuring the width of the intensity dip via the sweep of the standing wave phase. Finally, the spatial dimension of the sample can be measured from the width of the intensity dip if the other parameters are known. It should be noted that, in contrast to the precision position detection, these measurements relying on the determination of the width of the intensity dip are relative measurements in the sense that they do not require a reference to obtain the absolute standing wave phase. Only the change of the phase is relevant, and thus also knowledge of the actual position of the sample within the standing wave field is not required for these relative measurements. The maximum attenuation of the fluorescence intensity depends on the width of the collection relative to the width of the dip in the intensity profile. If the profile dip is narrower than the sample, then the dip only affects part of the sample. Still, this will result in a sudden reduction of the fluorescence intensity, which is sufficient to determine the onset of the overlap of intensity dip and atom sample. Also, a continuous scan of the standing wave phase results in repeated intensity dips over time which can be used to suppress statistical errors in the measurement.

\section{Single-pass localization}

In this section, we discuss a different experimental setup. We now assume that a collection of atoms (atomic cluster) flies through a standing wave field at an unknown position on the standing wave field axis. The aim is to gain as much information on the position as possible by measuring the collective resonance fluorescence. Obviously, the scanningdip scheme described in Sec. III B is not suitable for this kind of setup, since the change of the standing wave phase is too slow as compared to the interaction time of field and atom ensemble. In the present scheme, we only have to require that the time of flight $\tau_{f}$ through the standing wave field is much larger than the time $\tau_{s}$ needed to evolve into the steady state. For example, for a thermal beam with velocity a)

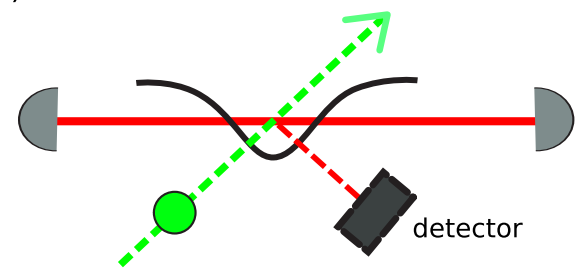

b)

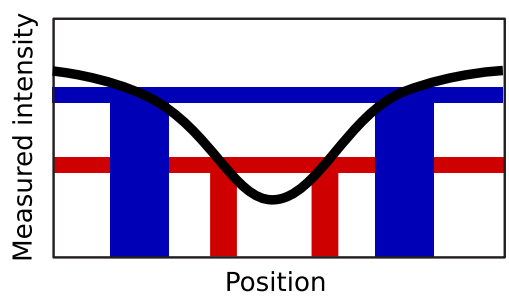

FIG. 6. (Color online) Single-pass localization. (a) shows the schematic setup. An ensemble indicated by the green dot passes through a standing wave field. The intensity of the scattered fluorescence light is measured. (b) Via the fluorescence intensity profile, the measured intensity can be related to the subwavelength particle position. The higher the slope of the intensity profile, the better is the accuracy of the localization. In this figure, the horizontal bars indicate two measurement outcomes with a certain uncertainty. The vertical bars show the corresponding potential positions. The black curve is a fluorescence intensity profile as, e.g., shown in Fig. 1.

$300 \mathrm{~m} / \mathrm{s}$ and a standing wave field width of $1 \mathrm{~mm}$, the flight time is about $\tau_{f}=3 \times 10^{-6} \mathrm{~s}$. The steady-state time $\tau_{s}$ is of the order $(N \gamma)^{-1}$. For $\gamma=10 \mathrm{MHz}$ and $N=10$, one obtains $\tau_{s}$ $=10^{-8} \mathrm{~s}$ and thus $\tau_{s} \ll \tau_{f}[21]$. Note that the preparation of atom clusters has been discussed in [22].

We now make use of the fact that position information can be gained from the absolute value of the scattered light intensity during the flight of the ensemble through the field. The schematic setup is shown in Fig. 6(a). The measured intensity allows one to fix a horizontal section in a collective fluorescence intensity plot versus ensemble position as shown in Fig. 6(b). Ideally, this section provides a set of few discrete points where the intensity profile crosses the measured intensity. These points correspond to the potential positions of the ensemble. From the two examples in Fig. 6(b) it is clear that the localization for a given intensity measurement with a measurement uncertainty becomes better with increasing slope of the intensity profile. A high slope, however, leads to a pronounced plateau with almost constant intensity in between the dips. This means that, in the case of a narrow dip, for large parts of the single wavelength width only a rather inaccurate localization is possible. Therefore, in contrast to the sweep scheme in Sec. III B, in this setup a wide intensity dip is desirable in order to achieve subwavelength localization for all possible positions. The reason is that for a wide intensity dip, wide plateaus in the intensity profile are avoided. A wide intensity dip can be achieved, for example, by working with weaker standing wave fields or far away from the symmetric collective-state resonance. 


\section{DISCUSSION AND SUMMARY}

We have described a scheme to localize small atomic samples with subwavelength accuracy. The scheme relies on measuring the superfluorescence radiation scattered in a standing wave field. We have demonstrated that external parameters such as the strength of the applied lasers or the detuning from the atomic resonance are convenient tools to tailor the localization region for a given experimental setup. Based on these results, two possible experimental situations have been considered. First, for fixed samples, a scanning- dip spectroscopy was proposed. Here, the standing wave field phase is changed in order to scan the fluorescence intensity profile along the cavity axis in order to reveal the actual position of the sample. This setup also allows for a number of relative measurements: for example, of distance between two collections, of the number of atoms in a sample, or of the linear dimension of the sample. Second, for samples passing through the standing wave field only once, a singlepass scheme was discussed, which relates the maximum intensity measured to the passing position of the sample. Our scheme can be generalized to the multiphoton case.
[1] R. Quadt, M. Collett, and D. F. Walls, Phys. Rev. Lett. 74, 351 (1995).

[2] J. E. Thomas and L. J. Wang, Phys. Rep. 262, 311 (1995).

[3] M. Holland, S. Marksteiner, P. Marte, and P. Zoller, Phys. Rev. Lett. 76, 3683 (1996)

[4] S. Kunze, K. Dieckmann, and G. Rempe, Phys. Rev. Lett. 78, 2038 (1997).

[5] S. Qamar, S.-Y. Zhu, and M. S. Zubairy, Phys. Rev. A 61, 063806 (2000); K. T. Kapale, S. Qamar, and M. S. Zubairy, ibid. 67, 023805 (2003); M. Sahrai, H. Tajalli, K. T. Kapale, and M. S. Zubairy, ibid. 72, 013820 (2005).

[6] E. Paspalakis and P. L. Knight, Phys. Rev. A 63, 065802 (2001); G. Liu, S. Gong, D. Cheng, X. Fan, and Z. Xu, ibid. 73, 025801 (2006).

[7] G. S. Agarwal and K. T. Kapale, J. Phys. B 39, 3437 (2006).

[8] P. Storey, M. Collett, and D. Walls, Phys. Rev. A 47, 405 (1993).

[9] C. Hettich, C. Schmitt, S. Zitzmann, S. Kühn, I. Gerhardt, and V. Sandoghder, Science 298, 385 (2002).

[10] M. D. Barnes, P. S. Krstic, P. Kumar, A. Mehta, and J. C. Wells, Phys. Rev. B 71, 241303(R) (2005).

[11] L. Zheng, C. Li, Y. Li, and C. P. Sun, Phys. Rev. A 71, 062101 (2005).

[12] J.-T. Chang, J. Evers, M. O. Scully, and M. S. Zubairy, Phys. Rev. A 73, 031803(R) (2006); J.-T. Chang, J. Evers, and M. S. Zubairy, ibid. 74, 043820 (2006).
[13] A. N. Boto, P. Kok, D. S. Abrams, S. L. Braunstein, C. P. Williams, and J. P. Dowling, Phys. Rev. Lett. 85, 2733 (2000); M. D’Angelo, M. V. Chekhova, and Y. Shih, ibid. 87, 013602 (2001); J. Xiong, D. Z. Cao, F. Huang, H. G. Li, X. J. Sun, and K. Wang, ibid. 94, 173601 (2005); C. H. Keitel and S. X. Hu, Appl. Phys. Lett. 80, 541 (2002).

[14] P. R. Hemmer, A. Muthukrishnan, M. O. Scully, and M. S. Zubairy, Phys. Rev. Lett. 96, 163603 (2006).

[15] G. S. Agarwal, Quantum Statistical Theories of Spontaneous Emission and their Relation to other Approaches (Springer, Berlin, 1974).

[16] Z. Ficek and S. Swain, Quantum Interference and Coherence: Theory and Experiments (Springer, Berlin, 2005).

[17] S. Ya. Kilin, Sov. Phys. JETP 55, 38 (1982).

[18] R. R. Puri, Mathematical Methods of Quantum Optics (Springer, Berlin, 2001) (especially Chap. 12 and references therein).

[19] G. Lenz and P. Meystre, Phys. Rev. A 48, 3365 (1993).

[20] M. Macovei, Z. Ficek, and C. H. Keitel, Phys. Rev. A 73, 063821 (2006).

[21] F. Schuda, C. R. Stroud, Jr., and M. Hercher, J. Phys. B 7, L198 (1974); J. M. Raimond, P. Goy, M. Gross, C. Fabre, and S. Haroche, Phys. Rev. Lett. 49, 1924 (1982); S. Kunze, S. Dürr, and G. Rempe, Europhys. Lett. 34, 343 (1996).

[22] G. M. D'Ariano, N. Sterpi, and A. Zucchetti, Phys. Rev. Lett. 74, 900 (1995). 